\title{
A clinical and radiological study in patients with idiopathic intracranial hypertension
}

\author{
Lubna Ibrahim Sultan ${ }^{1}$, Abdelaziz Mohamed Elnekidy ${ }^{2}$, Amr Mohamed Elfatatry ${ }^{1}$ and Amira Sayed ${ }^{1,3^{*}}$ (D)
}

\begin{abstract}
Idiopathic intracranial hypertension $(\mathrm{IIH})$ is one of the chronic causes of elevated intracranial pressure with no evident cause in neuroimaging and normal CSF analysis. It primarily affects overweight women of childbearing age.
\end{abstract}

Aim of work: To describe the clinical picture of $\mathrm{IH}$, neuroimaging and response to treatment after 3 months follow up in a sample of Egyptian patients.

Patients and methods: This was a prospective study carried out on 25 patients presented at Alexandria University Hospital with symptomatology of $\mathrm{IH}$. All the patients were subjected to complete history taking, neurological examination, fundus examination, lumbar puncture, and MRI brain and MR cerebral venography. The patients were followed-up for 3 months for assessment of treatment response.

Results: Patients' ages ranged from 1 0to 50 years, with a mean BMl $32.32 \pm 5.28 \mathrm{~kg} \mathrm{~m}^{2}$. Headache was the presenting manifestation in $100 \%$ of the patients. About $60 \%$ of the sample were either misdiagnosed or had a delayed diagnosis. There was a significant positive correlation between CSF opening pressure $(r=0.504, p=0.010)$, severity of headache $(r=0.472, p=0.017)$ and grade of papilledema. Optic hydropes and empty sellaturcica were the most common MRI abnormalities occurring in $95.8 \%$ and $70.8 \%$, respectively. Only $30 \%$ of the cases had normal MRV. Stenosis at genu junction and focal stenosis at transverse sinus occurred in $24 \%$ and $20 \%$ of the cases, respectively. Combined medical and serial lumbar puncture were the most effective line of therapy in the recruited patients.

Conclusion: Headache is the most common presentation of $\mathrm{IH}$, and its severity is positively correlated with papilledema grade. CSF pressure is also positively correlated with papilledema grade.

Keywords: $I H$, Headache, Papilledema, CSF, MRI

\section{Introduction}

Idiopathic intracranial hypertension (IIH) is one of the chronic causes of elevated intracranial pressure with normal cerebrospinal fluid (CSF) analysis and without any evident cause in neuroimaging [1]. There are several proposed mechanisms for IIH such as parenchymal edema, increased cerebral blood volume, excessive cerebrospinal fluid (CSF) production, venous outflow obstruction, and compromised CSF resorption. Secondary

\footnotetext{
*Correspondence: amirasiam12989@gmail.com

'Department of Neurology and Psychiatry, Faculty of Medicine, Alexandria University, Alexandria, Egypt

${ }^{3}$ Elhadara University Hospital, Moharram Bik, Alexandria, Egypt

Full list of author information is available at the end of the article
}

causes may lead to elevation of intracranial pressure (ICP) by different mechanisms from truly idiopathic causes [1]. IIH mainly affects obese females in childbearing period with incidence $12 / 100000$ per year. It can also occur in children, men, and non-obese persons [2].

Headache is the most common presentation in most of patients with IIH. Headache profile of IIH is not specific as it mimics the primary headaches with variable severity and duration. Patients present with symptoms as diplopia, blurred vision, transient visual obscurations, tinnitus, and visual loss. Other symptoms such as cervical and back pain, dizziness, nausea, and vomiting may also be present [3].

\section{Springer Open}

(- The Author(s). 2020 Open Access This article is licensed under a Creative Commons Attribution 4.0 International License, which permits use, sharing, adaptation, distribution and reproduction in any medium or format, as long as you give appropriate credit to the original author(s) and the source, provide a link to the Creative Commons licence, and indicate if changes were made. The images or other third party material in this article are included in the article's Creative Commons licence, unless indicated otherwise in a credit line to the material. If material is not included in the article's Creative Commons licence and your intended use is not permitted by statutory regulation or exceeds the permitted use, you will need to obtain permission directly from the copyright holder. To view a copy of this licence, visit http://creativecommons.org/licenses/by/4.0/. 
Papilledema is the most important cardinal sign for diagnosis of IIH. In the vast majority of the patients, the papilledema is symmetrical. Even though, asymmetrical papilledema was reported in a minority of patients. Moreover, the diagnosis of IIH can be made with other criteria even in the absence of edema. CSF opening pressure should be higher $25 \mathrm{cmH}_{2} \mathrm{O}$ for diagnosis of $\mathrm{IIH}$ according to the modified Dandy criteria with normal component of CSF [4].

Neuroimaging is essential for exclusion of other causes of increased intracranial pressure such as tumors, infections, and venous sinus thrombosis. Various signs of increased intracranial tension can be visualized on magnetic resonance imaging (MRI) such as distension of peri-optic nerve space, vertical touristy of optic nerve, intraocular protrusion of optic nerve, empty sellaturcica, enlarged Meckel's cave, flattening of posterior sclera, and posterior displacement of pituitary stalk [5]. On magnetic resonance venography (MRV), bilateral or unilateral transverse sinus stenosis (TSS) or sigmoidtransverse junction stenosis are common findings in patients with IIH. Other venous abnormalities include sigmoid sinus or internal jugular stenosis. MRV may be also normal [6].

Management of IIH includes weight loss, medical therapy (as acetazolamide, diuretics, or corticosteroids), venous sinus stenting and surgical procedures (as lumbo-peritoneal shunts, ventriculo-peritoneal shunts, or optic nerve fenestration). Surgical interventions are reserved for patients with fulminant IIH symptoms, e.g., rapidly progressive visual loss or severe intractable headache not responding to medical therapy [7].

The following are the aims of the present work:

1- Describe the demographic, health characteristics, and clinical picture of idiopathic intracranial hypertension

2- Identify intracranial vascular pathology of studied patients based on the used radiological studies (MRI and MRV)

3- Identify effectiveness of treatment regimens used in patients with intracranial hypertension after 3 months follow up

\section{Patients and methods}

This was a prospective cohort study conducted in Alexandria University Hospital during the period 20162018. Twenty five patients aged from 10 to 50 years were recruited to this study. All patients presented to the outpatient clinics with symptoms of idiopathic intracranial hypertension according to the modified Dandy criteria were included [8]. Patients who had space occupying lesions, venous sinus thrombosis, meningitis, ICP within normal range, abnormal CSF biochemistry or microbiology, or other causes of bilateral disk edema, e.g., ischemic optic neuropathy or diabetic papillopathy were excluded from the study. IIH patients without headache and IIH patients without papilledema were not included in this study.

All patients were subjected to complete history taking (including age, sex, headache onset, headache duration, frequency, site, character, severity, and intensity, associated symptoms, e.g., nausea, vomiting, photophobia, and phonophobia, tinnitus, visual complaints, e.g., blurring of vision, visual obscurations, diplopia, and visual loss). The headache intensity was evaluated by self-assessment of the patients using numerical rating scale (NRS). Patients reported the severity of headache on a scale from 0 to 10 [9]. Patients with scores from 1 to 3 were considered as mild headache, scores from 4 to 7 were considered moderate and scores from 7 to 10 were considered as severe headache. Past drug history were also revised with a special focus on hormonal contraception. All patients were subjected to routine laboratory chemistry, hemoglobin, and thyroid functions. Body mass index was calculated and complete neurological examination was performed for all patients. Fundus examination was done to assess the presence and grade papilledema using modified Frisén scale [10].

Lumbar puncture was performed and CSF opening pressure was measured using manometer in lateral decubitus position with the patients legs relaxed and partially extended. CSF chemistry and cytology were performed to ensure normality. MRI brain and posterior fossa, MRV or time of flight (TOF) were performed to exclude intracranial causes of elevated intracranial pressure, cerebral venous thrombosis. Patients were followed up for 3 months regarding clinical improvement of symptoms of headache severity, frequency, visual symptoms, and fundus examination (modified Frisén scale) to assess resolution of papilledema to assess treatment response. Patients were given either medical therapy, e.g., acetazolamide or diuretics (mild symptoms), combined medical therapy, and serial lumbar puncture (moderate symptoms), orlumbo-peritoneal shunt surgery (severe symptoms or intolerance to other treatment). Mild headache 1-3 at headache severity scale, moderate symptoms included headache from 4-6, severe headache more than 6 at headache scale (NRS).

Concerning the sample size was calculated by using the Epi info 7 program using the following formula:

$$
\mathrm{n}=\frac{\mathrm{t}^{2} \mathrm{X}(1-\mathrm{p})}{\mathrm{m}^{2}}
$$

where $n$ is the required sample size, $t$ is the confidence level at 95\% (standard value of 1.96), $p$ is the expected prevalence of idiopathic intracranial hypertension 1.5\% 
[11], $m$ is the margin of error at $5 \%$ (standard value of $0.05)$.

The expected prevelance of $\mathrm{IIH}$ of $1.5 \%$, a study power of $80 \%$ at $95 \%$ confidence level and a confidence limit of $5 \%$ based on this data, the total minimum appropriate sample size was estimated to be 23 patients.

All data were collected and analyzed using the IBM SPSS software package version 20.0 (Armonk, NY: IBM Corp). Qualitative data were described using number and percent. The normality of distribution was done using Kolmogorov-Smirnov test. Range (minimum and maximum), mean, standard deviation, and median were used to describe quantitative data. Significance of results was calculated at the $5 \%$ level. Chi-square test was used for categorical variables for comparison of different groups. Monte Carlo correction was used when chisquare results more than $20 \%$ of the cells have expected count less than 5 . Student $t$ test and Mann-Whitney test were used for normally and abnormally distributed quantitative variables respectively to compare between two studied groups. Kruskal-Wallis test was used for abnormally distributed quantitative variables, to compare between more than two studied groups, and post hoc (Dunn's multiple comparisons test) for pairwise comparisons. Pearson coefficient was used for correlation between two normally distributed quantitative variables, while Spearman coefficient was used for correlation between two distributed abnormally quantitative variables.

\section{Results}

The study was conducted on 25 female patients. The mean age of the patients was $27.84 \pm 10.47$ years with a range from 10 to 50 years. Sixty-four percent of patients were less than 30 years. The mean body mass index (BMI) was $32.32 \pm 5.28 \mathrm{~kg} \cdot \mathrm{m}^{2}$ with a range from 23.50 to $42.90 \mathrm{~kg} \mathrm{~m}^{2}$. Sixty-eight percent of the patients had BMI $>30 \mathrm{~kg} \mathrm{~m}^{2}$. The BMI was not significantly correlated with baseline fundus examination $(p$ value $=0.1)$, CSF opening pressure $(p$ value $=0.4$ ), or severity of headache $(p$ value $=0.1)$

\section{Clinical characteristics of the patients}

All patients $(100 \%)$ were presented by headache. Sixty percent of patients $(n=15)$ had continuous headache that occurred on daily basis, 7 patients $(28 \%)$ were presented by intermittent chronic headache (more than 15 headache days monthly), and 3 patients (12\%) had episodic headache that occurred less than 15 days monthly. Eleven patients $(44 \%)$ presented with bi-temporal headache, 6 patients $(24 \%)$ presented by global headache, 4 patients $(16 \%)$ presented by unilateral temporal headache, occipital headache in 2 patients (8\%), and both occipito-frontal headache in 2 patients $(8 \%)$.
Regarding the character of the headache, throbbing type was the most common character of the studied patients and was found in 18 patients (72\%), tension like headache in 4 patients (16\%), dull aching in 2 patients $(8 \%)$, and one patient had headache of unclassified character. Fourteen patients (56\%) described their headache as severe, 7 patients (28\%) reported moderate headache severity and 4 patients (16\%) reported headache of mild severity. Clinical severity of headache was significantly correlated with baseline fundus examination before treatment $(p$ value $=0.017)$.

Fourteen patients (56\%) reported associated symptoms to headache as nausea in 14 patients $(56 \%)$, vomiting in $6(24 \%)$, photophobia in 8 (32\%), and phonophobia in 2 (8\%). Median time from first symptoms to diagnosis was 60.0 days with range of duration from 0.0 to 1765.0 days. Only five patients sought medical advice without delay, while 20 patients $(80 \%)$ were delayed in diagnosis due to multiple causes. Negligence of symptoms was the most common cause of these causes, encountered in $10 \mathrm{pa}-$ tients (40\%). Three patients were misdiagnosed as errors of refraction, 2 patients were misdiagnosed as anemia, 2 patients were misdiagnosed as psychogenic headache, and 3 patients were misdiagnosed as other diagnoses as otitis media, general fatigue, and others.

Blurring of vision was found in 24 patients (96\%), diplopia was stated by 11 patients (44\%), visual obscurations were reported by 9 patients (36\%), and visual loss in 3 patients (12\%). Five patients (20\%) reported associated cervical and back pain, and one patient reported associated dizziness and cervical pain. Tinnitus was described in 14 patients (56\%) either unilateral or bilateral (Fig. 1), and was not correlated with CSF opening pressure or severity of headache.

Eleven patients (44\%) reported use of hormonal contraception (44\%), and the use was not significantly correlated with severity of headache ( $p$ value $=0.62$ ).

Sixty four percent of patients had normal neurological examination. Abducent nerve palsy was encountered in only 9 patients (36\%) of the recruited sample. Baseline fundus examination was done before starting therapeutic intervention. None of the recruited patients had a normal fundus. Symmetrical papilledema was found in 21 patients (84\%) while asymmetrical papilledema was found in 4 patients (16\%). After treatment and a 3month follow-up, 13 patients (52\%) had their papilledema totally resolved (i.e., normal fundus examination), 10 patients had partial improvement of their papilledema grade and only 2 patients had residual high grades of papilledema (GIII-GV) as shown in Table 1. There was no correlation between papilledema grades before and after treatment ( $p$ value $=0.16$ ). There was also no statistically significant correlation between follow-up grade of papilledema and BMI of the patients ( $p$ value $=$ 


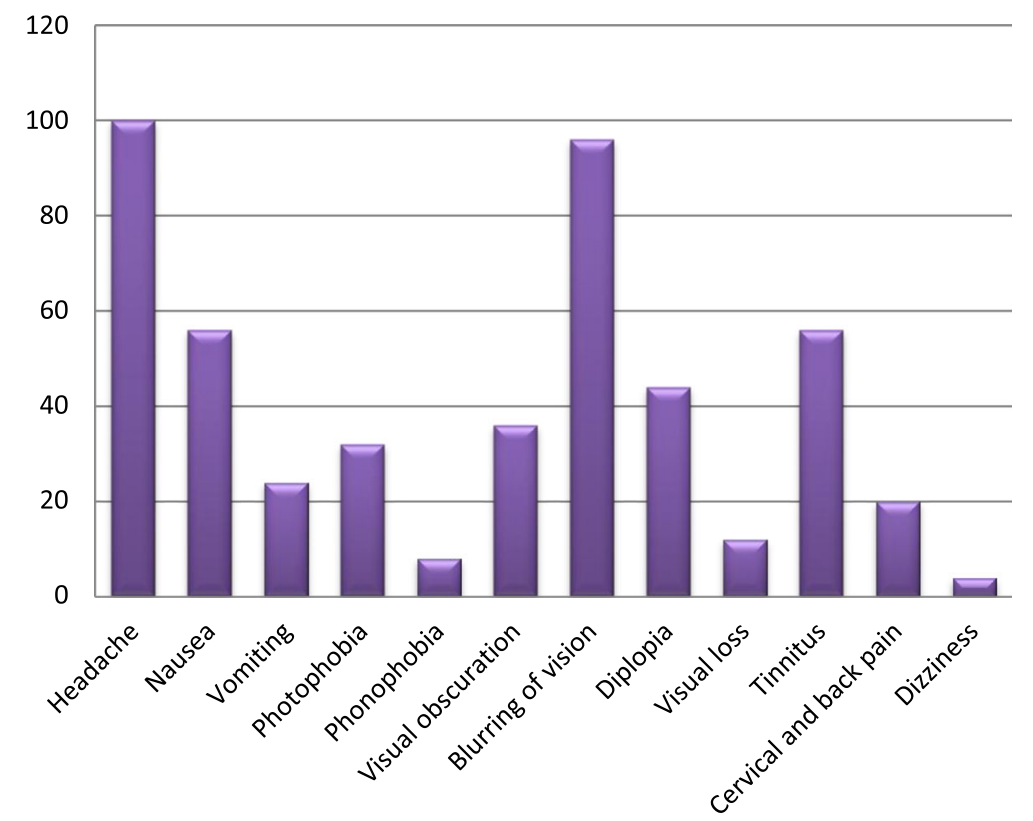

Fig. 1 Distribution of patients according to symptoms $(n=25)$

0.297). The grade of papilledema was only significantly correlated with the severity of headache $(p=0.017)$.

With regards to the laboratory profile of the studied patients, routine chemistry panel was unremarkable in all patients. Eight patients had anemia. The median $\mathrm{Hb}$ level was $12.60 \mathrm{~g} / \mathrm{dl}(10.50-15.0 \mathrm{~g} / \mathrm{dl})$. One patient had hypothyroid and started l-thyroxin therapy. The median CSF opening pressure was $39.0 \mathrm{~cm} \mathrm{H} 2 \mathrm{o}$ (25.0 to 80.0 $\mathrm{cmH}_{2} \mathrm{O}$ ). The CSF opening pressure was positively correlated with both baseline fundus examination $(p=0.010)$ and severity of headache $(p=0.045)$ (Figs. 2 and 3$)$ and

Table 1 Distribution of the studied cases according to fundus examination $(n=25)$

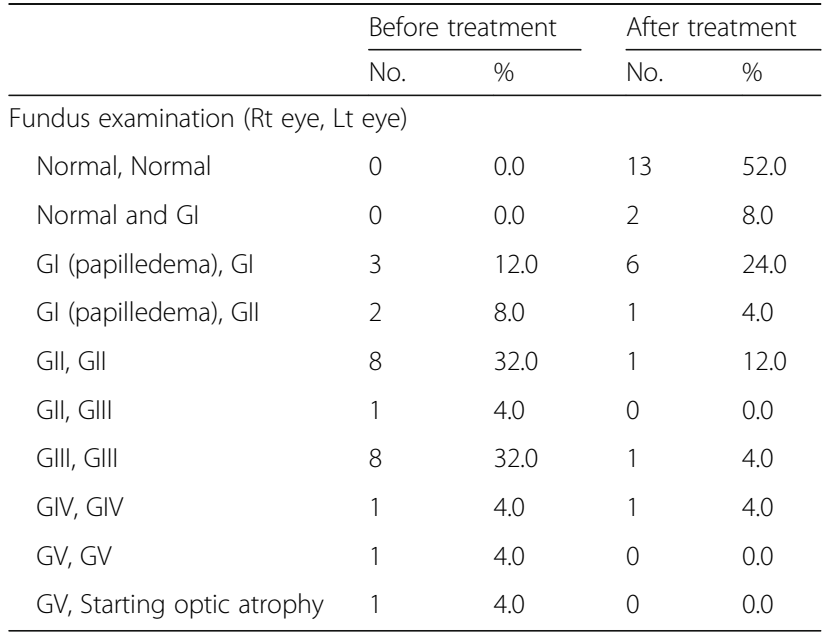

inversely correlated with age of the patient $(r=-0.43, p$ $=0.03$ ).

On reviewing the patient's imaging, the MRI brain abnormalities seen among the studies sample were optic hydropes (optic nerve sheath distension) in 17 patient (70\%), empty sellaturcica in 23 patient (95\%), prominent Meckel's cave in 16 patient (66.7\%), flattening of posterior sclera in 12 patients (50\%), vertical tortuosity of optic

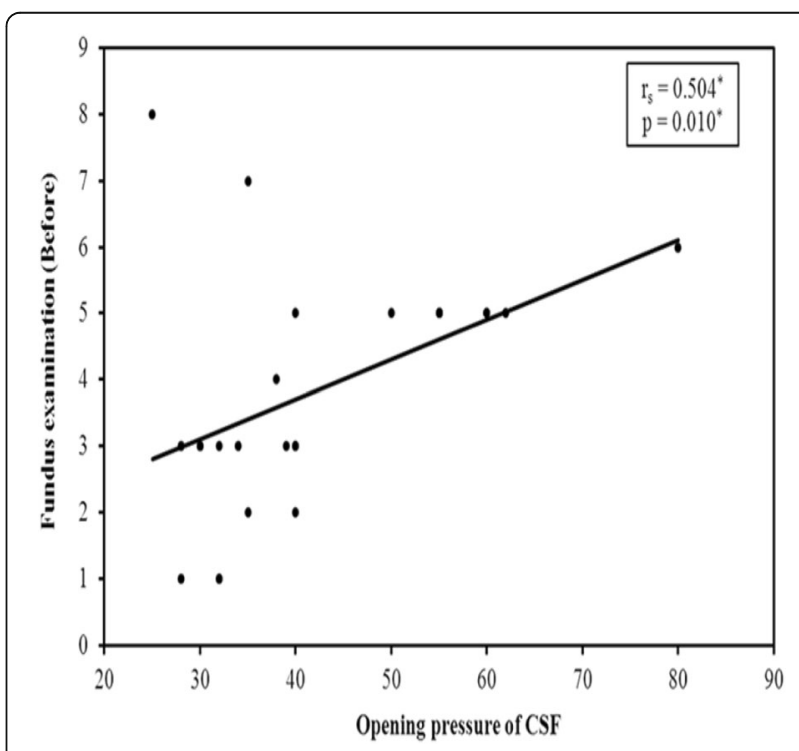

Fig. 2 Correlation between opening pressure of CSF with fundus examination (before) $(n=25)$ 


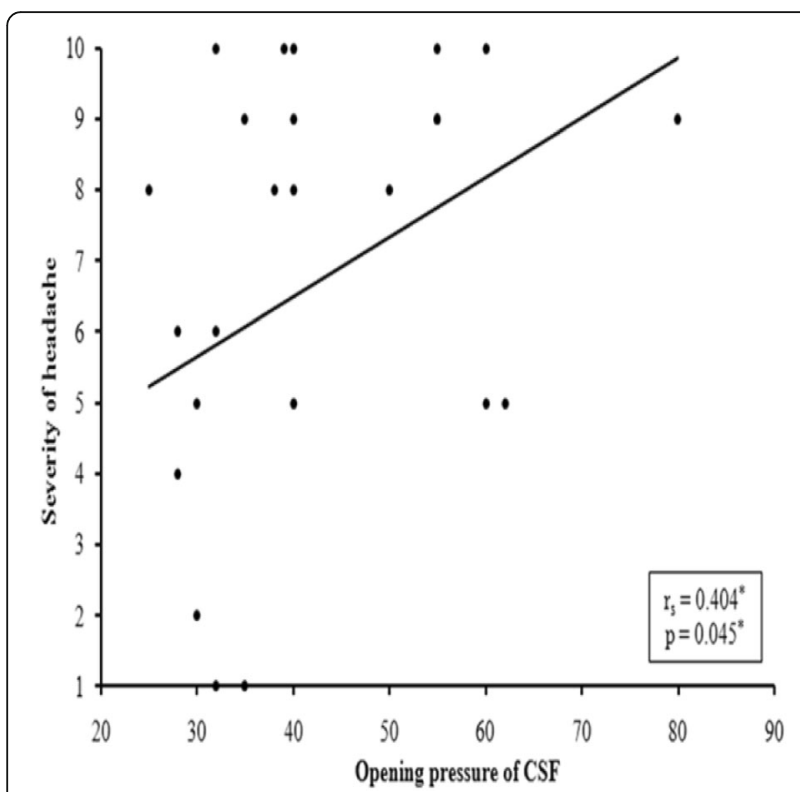

Fig. 3 Correlation between opening pressure of CSF with severity of headache $(n=25)$

nerve in 15 patients (62.5\%), intraocular protrusion of optic nerve in 6 patients, and posterior displacement of the pituitary stalk in 6 patients. Individual imaging findings are summarized in Table 2 and demonstrated in Figs. 4, 5, 6, and 7. The presence of vertical tortuosity of the optic nerve was positively correlated with the CSF opening pressure ( $p$ value $=0.039)$. The grading of sellaturcica emptying seen in our patients is depicted in Table 3. The grade of sellaturica emptying was not found to be correlated with neither the CSF opening pressure $(p=0.351)$ nor the duration of headache $(p=$ $0.52)$.

MR venography was normal in only 8 patients. Six patients had transverse sinus hypoplasia, 5 patients had bilateral genu stenosis and 3 patients had both findings. Less frequently seen abnormalities were focal transverse

Table 2 Distribution of the studied cases according to MRI brain $(n=25)$

\begin{tabular}{lll}
\hline MRI brain & No. & \% \\
\hline Normal & 1 & 4.0 \\
Abnormal & $\mathbf{2 4}$ & $\mathbf{9 6 . 0}$ \\
$\quad$ Optic hydropes & 17 & 70.8 \\
Flattening of post sclera & 12 & 50.0 \\
Prominent Meckel's cave & 16 & 66.7 \\
Intraocular protrusion of optic nerve & 6 & 25.0 \\
Displacement of posterior stalk & 6 & 25.0 \\
Empty sellaturcica & 23 & 95.8 \\
Vertical tortuosity of optic nerve & 15 & 62.5 \\
\hline
\end{tabular}

sinus stenotic area in a single patient, attenuated sigmoid in another, and genu stenosis with transverse and sigmoid sinus stenosis in a third patient (Table 4) (Fig. $8)$. None of the MRV findings were significantly correlated with either the CSF opening pressure ( $p$ value $=$ $0.7)$ or the response to treatment on follow-up. Even though, patients with abnormal MRV findings $(n=17)$ were significantly younger than their counterparts who had normal MRV $(p=0.03)$.

Concerning the treatment, only five patients were given and maintained on medical therapy only. acetazolamide (up to $2.5 \mathrm{~g}$ daily) and furosemide (20-40 mg daily) were the medications used. Combined medical therapy and serial lumbar puncture was utilized in 17 patients (68\%). Three patients required CSF diversion procedures such as lumbo-peritoneal shunt, but none of them had stenting of venous sinus.

After 3-month follow-up, 17 patients (68\%) showed total improvement of their headache and visual symptoms. Six patients reported partial improvement, and only 2 patients did not show any improvement and were indicated for surgery. Of the five patients who were given medical treatment alone, a single one reported total improvement of her symptoms $(p=0.008)$. The remaining four reported either partial $(n=2)$ or no improvement $(n=2)$. Of the 17 patients who received combined medical and serial lumbar puncture, 14 had total improvement of their symptoms $(p$ value $=0.031$ ) The remaining three reported partial improvement. Two out of the three patients who had shunt surgery had total improvement, and the third reported partial improvement. The response to treatment was not found to be significantly correlated to the CSF opening pressure $(p=0.4)$, the patient age $(p=0.22)$, the BMI $(p=0.2)$, the grade of papilledema $(p=0.18)$, or the previous duration of headache ( $p$ value $=0.6$ ).

\section{Discussion}

All our studied patients were obese females with high body mass index (BMI) (above $25 \mathrm{~kg} / \mathrm{m}^{2}$ ) as reported in previous studies which reported also that the BMI was not significantly correlated with neither CSF opening pressure nor clinical severity of headache as our study. We found that the CSF pressure tended to be higher in patients with higher BMI $[12,13]$. This lack of correlation between BMI and severity of headache may be attributed to the subjective assessment of pain and the different pain threshold among the participants.

IIH was also reported to occur in old age (such as 80 years); however, they present mainly with visual manifestation and mild headache. Their prognosis is usually better than young adults $[14,15]$. In contrast, Yri et al. [16] reported that young age and high initial ICP were associated with better headache outcome after 1 year of 


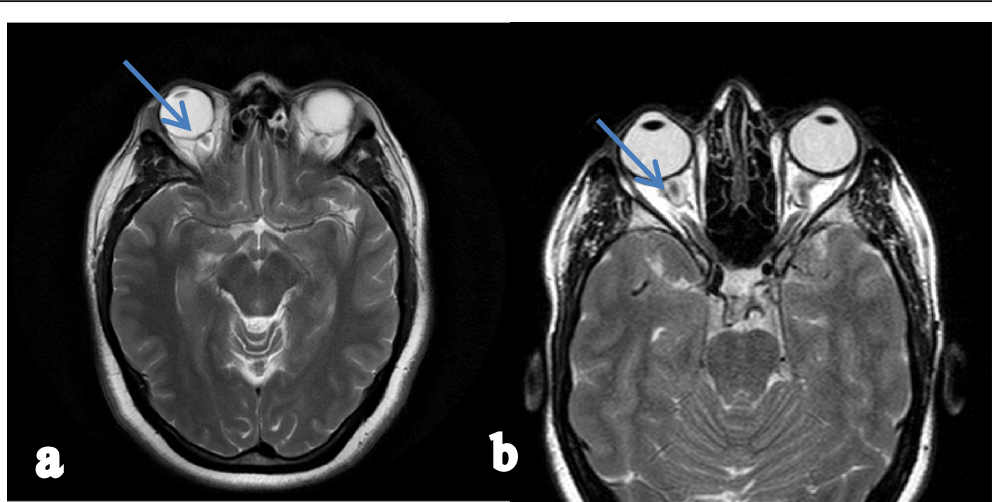

Fig. 4 MRI brain T2 sequence of a 25-year-old lady showing (a) intraocular protrusion of optic nerves and (b) optic hydrops

follow-up. The explanation for their finding was the different etiologies of IIH in young and old age [16], for example, IIH occurring in younger age (especially children) is mostly secondary to endocrinal disturbances, sinusitis, and prepubertal hormonal changes. However, the IIH occurring in adults is idiopathic and the exact cause are unknown. In the current study, there was no correlation between age and follow-up clinical response. This may be due to the short period of follow-up (3 months) in comparison to 1 year follow-up reported in the literature.

Multiple comorbidities and drug use commonly oral contraceptive pills were reported to be associated with IIH. Many studies found that patients with IIH commonly have comorbidities which may have a role in the

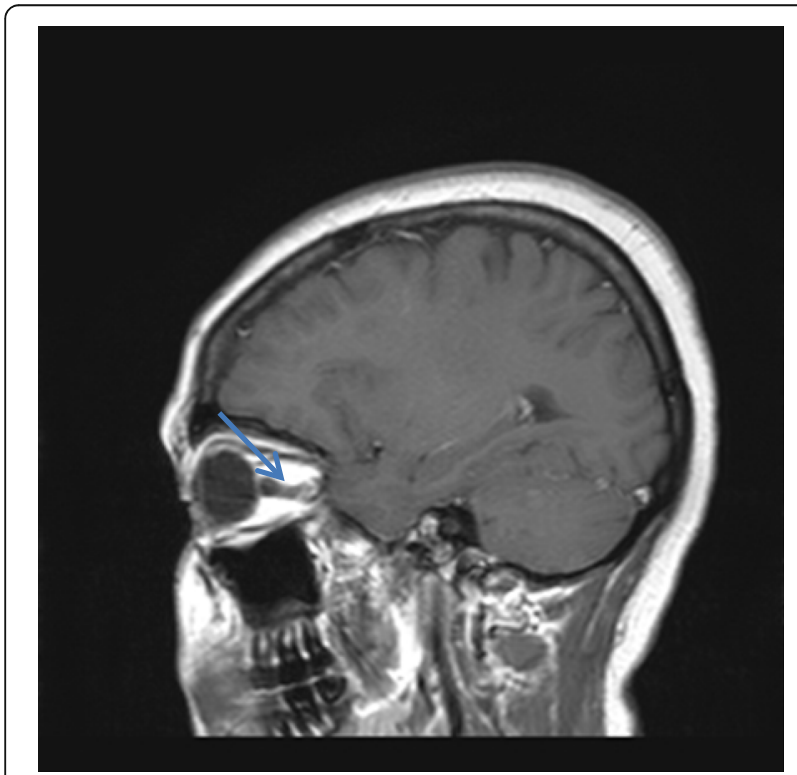

Fig. 5 MRI brain T1 sequence of 28-years-old lady showing vertical tortuosity of optic nerve pathogenesis of the disease such as polycystic ovary syndrome (PCO), anemia, endocrinal disorders as hypothyroidism. We found no correlation between use of hormonal contraception and the severity of headache in contrast to the findings of another study which reported a significant correlation between the use of hormonal contraception and severity of headache assessed by headache impact test [13]. This may be attributed to different headache scales used. Misdiagnosis and delayed diagnosis are common among IIH patients as reported in previous studies. Usually, patients neglect their headache when it is relieved by analgesics. They sought medical advice either due to increase severity of headache, development of diplopia, or decrease in visual acuity, thus indicating IIH diagnosis $[17,18]$.

Headache is the most common presentation in patients with IIH. However, the headache profile is not pathognomonic in IIH [19]. In previous IIH studies, headache character, site, and frequency varied among patients with the throbbing temporal headache constituting the most common type. Only few patients experienced headache of unclassified character [20, 21]. In agreement with previous studies, it was found in the current study that daily constant, bitemporal, throbbing, and severe headache was the most common type of headache encountered.

Transient visual obscurations are the second most common symptom described in patients with IIH in multiple studies $[12,22]$. Other studies, as well as this study, found that blurred vision and diplopia were the predominant visual symptoms. Tinnitus is experienced by many IIH patients which may be due to intensified vascular pulsation and flow disturbances in venous system.

Papilledema is considered the most important cardinal sign in this disease. In agreement with previous studies $[12,13,22]$, it was found that most of our patients $(84 \%)$ had symmetrical grades of papilledema, and only a 


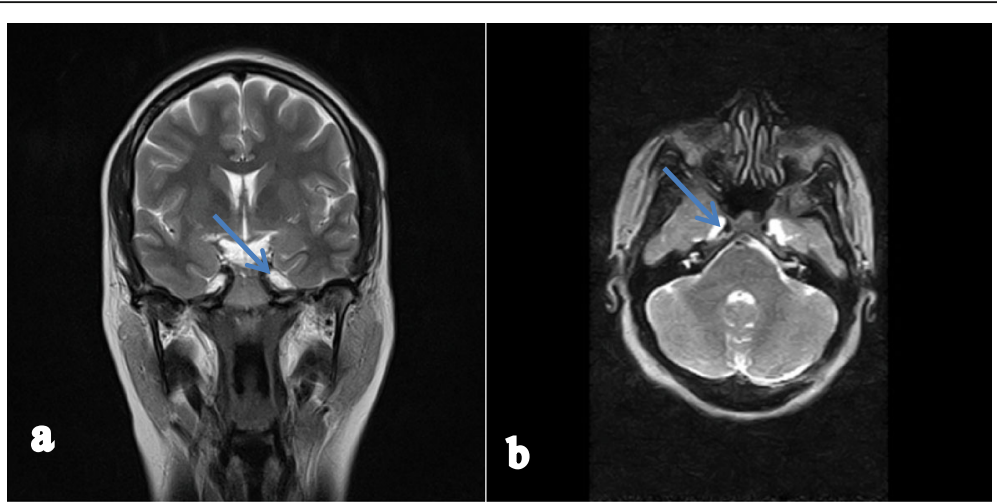

Fig. 6 MRI brain T2 sequence of a 31-year-old lady showing prominent Meckel caves in coronal (a) and axial (b) views

minority (16\%) showed asymmetrical grades. This asymmetry may be due to optic nerve sheath defects and loss of lamina cribrosa compliance. In agreement with previous studies [22, 23], papilledema grade was significantly correlated with CSF opening pressure and the severity of headache. This is in contrary with Friedman study that revealed no correlation between HIT-6 headache severity score and papilledema grade [17].

Previous studies proved the lack of correlation between CSF opening pressure and severity of headache using HIT-6 score $[17,22]$. The lack of association between the presence and intensity of headache and CSF

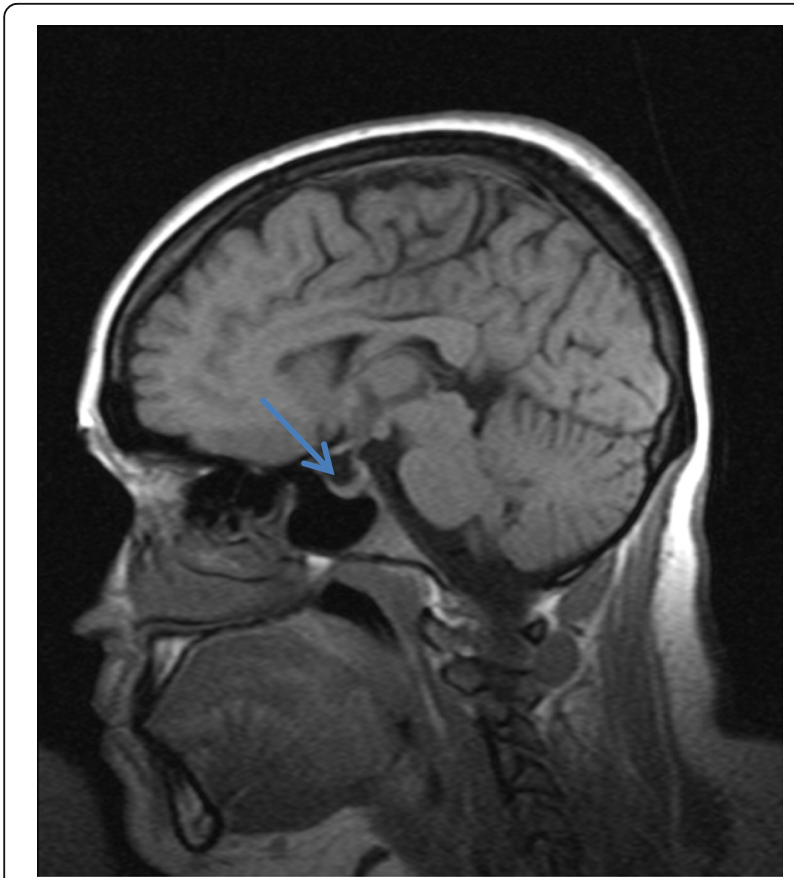

Fig. $7 \mathrm{MRI}$ brain T1 sequence sagittal view of a 24-year-old lady showing empty sella turcica grade IV and posterior displacement of pituitary stalk opening pressure may be a result of natural fluctuation in CSF pressure throughout the day [24]. In the current study, all patients with high CSF pressure had reported headache with a significant correlation between CSF opening pressure and severity of headache.

In the current study, MRI was normal in only one patient. It showed also separate or combined signs of increased ICP. Posterior globe flattening, optic nerve sheath distension, optic nerve tortuosity, and empty sellaturcica were the most common MRI findings. None of our cases had slit-like ventricles in their MRI as it is highly specific but least sensitive sign as previously proved [25]. Larger sample size may be needed for further detection of slit-like ventricles. Combination of radiological signs of IIH raises the specificity for the diagnosis of IIH.

These radiological signs were reported by Lim et al. [26], Agid et al. [27], and others [6, 27] who had described the specificity and sensitivity of these imaging findings in IIH. Those researchers reported that posterior globe flattening, optic nerve protrusion, and slit-like ventricles had maximum specificity (100\%). Empty or partially empty sella is a non-specific sign. It is present in normal individuals or in any cause of elevation in ICP [28], [6, 28]. In agreement with our study, another radiological markers that were revealed in our sample were prominent Meckel's cave and posterior displacement of pituitary stalk [25, 29]. Transverse sinus abnormalities either unilateral or bilateral were found to be common

Table 3 Distribution of the studied cases according to empty sella turcica grade $(n=23)$

\begin{tabular}{lll}
\hline Empty sella turcica & No. & $\%$ \\
\hline GI & 8 & 34.8 \\
GII & 4 & 17.4 \\
GIII & 4 & 17.4 \\
GIV & 7 & 30.4 \\
\hline
\end{tabular}


Table 4 Distribution of the studied cases according to MRV finding $(n=25)$

\begin{tabular}{lll}
\hline MRV finding & No. & $\%$ \\
\hline Normal & 8 & 32.0 \\
Hypoplasia of TS & 6 & 24.0 \\
Stenosis at genu junction (bilateral) & 5 & 20.0 \\
Focal stenotic area TS & 1 & 4.0 \\
Attenuated sigmoid & 1 & 4.0 \\
Stenosis at genu junction (Bilateral) and hypoplasia of TS & 3 & 12.0 \\
Stenosis at genu junction and stenosis of right TS, SS & 1 & 4.0 \\
\hline
\end{tabular}

in our study in agreement with previous studies [13, 25]. The majority of patients have normal finding suggesting that IIH may not be caused by venous cause or stenosis. In spite of the findings from another study that bilateral transverse venous sinus stenosis was present in absence of diagnosis of IIH. The elevated ICP may result in more worsening of TSS. In contrary to Chagot et al. who stated that bilateral TSS was significantly higher among older patients, we found that older patients had normal MRV. This difference may be due to the small size of our sample.

We found no significant improvement on medical therapy only. Unlike other previous studies that reported significant improvement among patients who received medical therapy and further significant improvement when weight loss was added [30]. This denotes that weight loss is an important line of treatment. The present study showed similar total improvement in most of patients who received combined medical and repeated lumbar puncture. In previous studies, repeated spinal tapping was mainly used in pregnant women as the best line of treatment and showed improvement in their symptoms [31].

In the present study, there was an improvement in papilledema grade after treatment in most of the patients. No correlation was found between papilledema grade before treatment in IIH and its improvement after treatment and follow-up. Previous studies showed that treatment effect was greater in patients with a higher papilledema grade at baseline compared to mild grade [22]. The response time for the optic disk to normalize after treatment may depend on the severity of baseline disk edema but, Maren Skau found that severity of papilledema at presentation is not predictive for chronic course of IIH [18]. Thin patients experienced high incidence of relapse of symptoms after 3 months follow-up after medical therapy and weight loss [32]. However, in disagreement with our study, morbid obesity (BMI > 40 $\mathrm{kg} \cdot \mathrm{m}^{-2}$ ) was demonstrated as a factor of poor visual outcome [15]. This difference can be attributed to the different methods used for visual assessment as we used fundus examination for evaluation of papilledema while used perimetry for evaluation of visual outcome.

Our study is the first study in Egypt describing the clinical and radiological criteria in IIH patients and their response o treatment after 3 months follow-up.

Our limitations in this study are short follow-up period, absence of male gender for comparison of results and no OCT or parametric studies done for patients.

\section{Conclusion}

Headache is the most common presentation in $\mathrm{IIH}$ patients with no specific character, site, frequency, or intensity. Blurring of vision, diplopia, and visual obscurations are common visual complains in patients with $\mathrm{IIH}$. Symmetrical papilledema is the most sign encountered on fundus examination. CSF opening pressure is correlated with grade of papilledema, severity of headache are inversely correlated with age of the patients. Transverse sinus abnormalities and sigmoid-transverse junction stenosis are common MRI signs for IIH. Combined medical therapy and repeated lumbar puncture are the most common line of treatment used in the recruited sample.

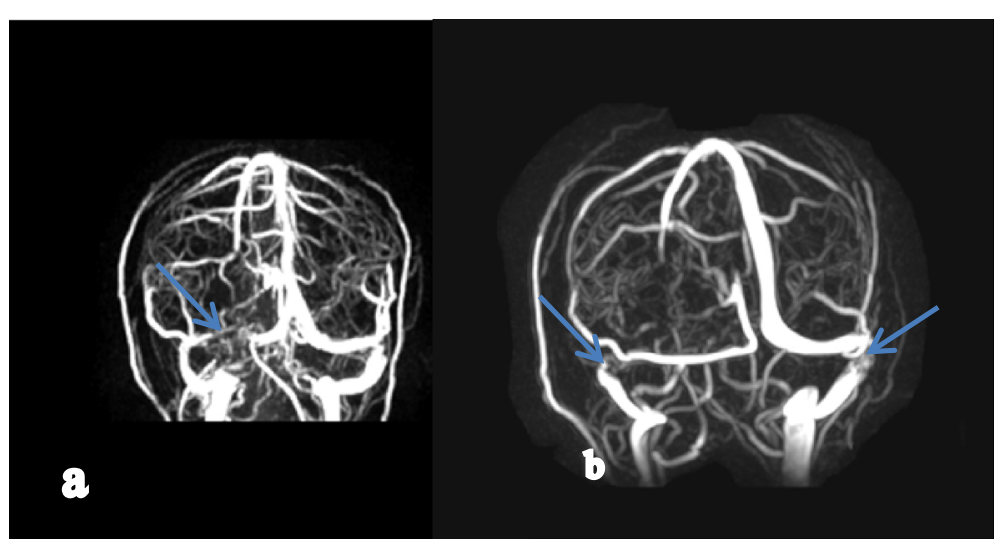

Fig. 8 a Attenuated transverse sinus, b MRV brain showing bilateral stenosis of transverse-sigmoid sinus with hypoplasia of the transverse sinus on the right side 


\section{Abbreviations}

BMl: Body mass index; BVP: Biventriculoperitoneal; COPD: Chronic obstructive pulmonary disease; CSF: Cerebrospinal fluid; HIT-6: Headache-impact test-6; IIH: Idiopathic intracranial hypertension; ICP: Intracranial pressure; MRI: Magnetic resonance imaging; MRV: Magnetic resonance venography; OCT: Optical coherence tomography; PCO: Polycystic ovary; PMD: Perimetric mean deviation; TSS: Transverse sinus stenosis; TOF: Time of flight

\section{Acknowledgements}

None to be mentioned

\section{Authors' contributions}

$\mathrm{LI}$ participated in creating the idea and principle of the conducted research and he recruited the cases for research. AM was the radiologist who revised radiological findings for all patients, supervised, and revised the written material created the study design, methodology, and revised the writing process. AM was the radiologist who revised radiological findings for all patients, supervised, and revised the written material. AS is the corresponding author. The authors read and approved the final manuscript.

\section{Funding}

None

\section{Availability of data and materials}

Available with manuscript.

\section{Ethics approval and consent to participate}

Consent was taken from all patients and the research was approved by the Ethics Committee of the Faculty of Medicine-Alexandria University (which is constituted and operates according to ICH GCP guidelines and applicable local and institutional regulations and guidelines which govern EC operation for the last 9 years. It has FWA from 2010). The ethical approval was taken on June 2016, and the reference number is 0104555

\section{Consent for publication}

All participants had signed an informed consent to participate and for the data to be published without names.

\section{Competing interests}

The authors declare that have no competing interests.

\section{Author details}

'Department of Neurology and Psychiatry, Faculty of Medicine, Alexandria University, Alexandria, Egypt. ${ }^{2}$ Department of Radiology, Faculty of Medicine, Alexandria University, Alexandria, Egypt. ${ }^{3}$ Elhadara University Hospital, Moharram Bik, Alexandria, Egypt.

Received: 23 June 2019 Accepted: 5 June 2020

Published online: 08 July 2020

References

1. Mollan SP, et al. Evolving evidence in adult idiopathic intracranial hypertension: pathophysiology and management. J Neurol Neurosurg Psychiatry. 2015;87(9):982-92.

2. Wakerley BR, Tan MH, Ting EY. Idiopathic intracranial hypertension. Cephalalgia. 2015;35(3):248-61.

3. Friedman DI. The pseudotumor cerebri syndrome. Neurol Clin. 2014:32(2): 363-96.

4. Bidot $\mathrm{S}$, et al. Asymmetric papilledema in idiopathic intracranial hypertension. J Neuroophthalmol. 2015;35(1):31-6.

5. Butros SR, et al. Imaging features of idiopathic intracranial hypertension, including a new finding: widening of the foramen ovale. Acta Radiol. 2012; 53(6):682-8.

6. Ridha MA, et al. MRI findings of elevated intracranial pressure in cerebral venous thrombosis versus idiopathic intracranial hypertension with transverse sinus stenosis. Neuroophthalmology. 2013;37(1):1-6.

7. Mollan SP, et al. A practical approach to, diagnosis, assessment and management of idiopathic intracranial hypertension. Pract Neurol. 2014; 14(6):380-90.

8. Friedman DI, Jacobson DM. Diagnostic criteria for idiopathic intracrania hypertension. Neurology. 2002;59(10):1492-5.
9. Pain: clinical manual for nursing practice Pain: clinical manual for nursing practice Margo McCaffery Alexander Beebe Mosby Yearbook UK pound17. 25072341992 2. Nurs Stand. 1994;9(11):55.

10. Scott CJ, et al. Diagnosis and grading of papilledema in patients with raised intracranial pressure using optical coherence tomography vs clinical expert assessment using a clinical staging scale. Arch Ophthalmol. 2010;128(6):705-11.

11. Kesler A, Gadoth N. Epidemiology of idiopathic intracranial hypertension in Israel. J Neuroophthalmol. 2001;21(1):12-4.

12. Galvin JA, Van Stavern GP. Clinical characterization of idiopathic intracranial hypertension at the Detroit Medical Center. J Neurol Sci. 2004;223(2):157-60.

13. Chagot $C$, et al. Idiopathic intracranial hypertension: prognostic factors and multidisciplinary management. J Obes. 2017;2017:5348928.

14. Demet Arslan AA, Akı E. Clinical and demographic features of pseudotumor cerebri syndrome diagnosed in a university hospital. Turk J Neurol. 2017;23: 60-3.

15. Bruce BB, et al. Atypical idiopathic intracranial hypertension: normal BMI and older patients. Neurology. 2010;74(22):1827-32.

16. Yri HM, et al. The course of headache in idiopathic intracranial hypertension: a 12-month prospective follow-up study. Eur J Neurol. 2014;21(12):1458-64.

17. Friedman Dl, et al. Headache in idiopathic intracranial hypertension: findings from the idiopathic intracranial hypertension treatment trial. Headache. 2017;57(8):1195-205

18. Skau M, et al. Disease activity in idiopathic intracranial hypertension: a 3month follow-up study. J Neurol. 2011;258(2):277-83.

19. Yri HM, et al. Idiopathic intracranial hypertension is not benign: a long-term outcome study. J Neurol. 2012;259(5):886-94.

20. Yri HM, Jensen $\mathrm{RH}$. Idiopathic intracranial hypertension: clinical nosography and field-testing of the ICHD diagnostic criteria. A case-control study. Cephalalgia. 2015;35(7):553-62.

21. Digre $\mathrm{KB}$, et al. A comparison of idiopathic intracranial hypertension with and without papilledema. Headache. 2009;49(2):185-93.

22. Smith SV, Friedman DI. The idiopathic intracranial hypertension treatment trial: a review of the outcomes. Headache. 2017;57(8):1303-10.

23. Pollak $L$, et al. Reevaluation of presentation and course of idiopathic intracranial hypertension--a large cohort comprehensive study. Acta Neurol Scand. 2013:127(6):406-12.

24. Johnston I, Paterson A. Benign intracranial hypertension. II. CSF pressure and circulation. Brain. 1974:97(2):301-12.

25. Maralani PJ, et al. Accuracy of brain imaging in the diagnosis of idiopathic intracranial hypertension. Clin Radiol. 2012;67(7):656-63.

26. Lim MJ, et al. Magnetic resonance imaging changes in idiopathic intracranial hypertension in children. J Child Neurol. 2010;25(3):294-9.

27. Agid $R$, et al. Idiopathic intracranial hypertension: the validity of crosssectional neuroimaging signs. Neuroradiology. 2006;48(8):521-7.

28. Saindane AM, et al. Association of MRI findings and visual outcome in idiopathic intracranial hypertension. AJR Am J Roentgenol. 2013;201(2): 412-8.

29. Aaron GP, et al. Enlargement of Meckel's cave in patients with spontaneous cerebrospinal fluid leaks. Int Forum Allergy Rhinol. 2017;7(4):421-4.

30. Piper RJ, et al. Interventions for idiopathic intracranial hypertension. Cochrane Database Syst Rev. 2015;8:CD003434.

31. Eissaa $E$, et al. Lumbar puncture as a single modality for treatment of idiopathic intracranial hypertension during pregnancy. Egypt J Neurol Psychiatry Neurosurg. 2016;53(1):33-6.

32. Gafoor VA, Smita B, Jose J. Long-term response of cerebrospinal fluid pressure in patients with idiopathic intracranial hypertension - a prospective observational study. Ann Indian Acad Neurol. 2017;20(3):220-4.

\section{Publisher's Note}

Springer Nature remains neutral with regard to jurisdictional claims in published maps and institutional affiliations. 